\title{
An RFLP-based genetic map of pearl millet (Pennisetum glaucum)
}

\begin{abstract}
Analysis of a sample of diverse pearl millet genotypes with 200 genomic DNA probes revealed this crop species to be extremely polymorphic. Among these genotypes, $85 \%$ of probes detected polymorphism using only two restriction enzymes, with an average pair-wise polymorphism between all of the probe-enzyme combinations of $56 \%$. Two crosses were employed to construct an RFLPbased genetic map. In an intervarietal $F_{2}$ population, derived from a single $F_{1}$ plant, 181 loci were placed on a linkage map. The total length of this map, which comprised seven linkage groups, was $303 \mathrm{cM}$ and the average map distance between loci was about $2 \mathrm{cM}$, although a few intervals in excess of $10 \mathrm{cM}$ were present at the ends of a few linkage groups. Very few clones, including those which hybridized to more than one copy, detected more than one locus in the pearl millet genome. The analysis was complicated initially because 83 of the 181 loci mapped to a single linkage group. Analysis of a second cross identified a probable translocation breakpoint in the middle of this large linkage group.
\end{abstract}

Key words Pearl millet - RFLP - Translocation Genetic maps

\section{Introduction}

Pearl millet [Pennisetum glaucum (L.) R. Br.; $2 \mathrm{n}=$ $2 x=14$ ] is an important crop providing the staple diet of

\section{Communicated by G. E. Hart}

C. J. Liu - T. S. Pitlaway - M. Nash - C. S. Busso - M. D. Gale (因) Cambridge Laboratory, Colney Lane, Norwich NR4 7UJ. UK

\section{J. R. Witcombe}

Centre for Arid Zone Studies, Bangor, Gwynedd LL57 2UW, Wales, UK

\section{T. Hash}

International Crops Research Institute for the Semi-Arid Tropics, Patancheru, AP 502 324, India people living in large areas of the semi-arid tropics of Africa and India. In terms of annual global production, pearl millet is the sixth most important cereal crop. following wheat, rice, maize, barley and sorghum (Brunken 1977). Although pearl millet has a remarkable ability to grow on soils of marginal fertility and semi-arid conditions, it responds very well to fertilization and irrigation (Minocha 1991 ). In addition, this species has also proved to be ideal for genetic study. Millet has a haploid (1C) DNA content of about $2.5 \mathrm{pg}$ (Bennett 1976) and possesses abundant phenotypic variation (Brunken 1977). Moreover, it has a number of wild relatives with haploid chromosome complements of 5,8 and 9, in addition to a large group with $2 n$ $=14$ with which it can be intercrossed (Jauhar 1968, 1981).

Despite its economic importance and amenability as a research organism, the genome of pearl millet has been little investigated. In this paper we report on the first detailed linkage map of $P$. glaucum.

\section{Materials and methods}

Genetic stocks

Genotypes. Nineteen genotypes were employed to assess the level of polymorphism in this crop species. They were Tift $23 \mathrm{DB}_{1}, 5141 \mathrm{~B}$. ICMB 841, 81B, 843B, J 104, H 77/833-2, ICME 423, ICMP 451, K 560, ICMH 423, ICMH 451, BK 560, HHB 67, 700651-1, P 7-3. 7042(S), ICMP 85410, and LGD-1-B-10. These genotypes were selected both because of their range of geographical origin and as potential parents of mapping populations.

Parents and $F_{2}$ populations. Two single crosses involving four parents were made. The first cross was between LGD-1-B-10 (C. $T$ Hash, unpublished), a derivative of (B70 $\times$ Tift 756)-1-4-5 and ICMP 85410 (ICRISAT, unpublished). This cross is referred to below as LGD $\times 85410$. The second cross was between Tift $23 \mathrm{DB}_{1}$ and WSIL (IP 18292). This cross is referred to as 23DB $\times$ WSIL. LGD $\times 85410$ was selected for mapping based on the analysis of the 19 genotypes with 200 low-copy probes. A population of $133 F_{2}$ plants was studied. When it became apparent that this cross may have involved an interchromosomal translocation, $132 \mathrm{~F}_{2}$ plants from the cross 23DB $\times$ WSIL were grown and used for transfer of a skeleton map from the linkage group with the suspected translocation event. 
DNA probes

Most clones were derived from a pearl millet $P_{s t} l$ genomic library. This library, containing 1000 clones (PgPSMI-PgPSM100)()), was constructed with total-plant DNA extracted from leaves of the pearl millet genotype 7042(S). Fifty mickigrams of DNA were digested to completion with 1(0) U PstI and subjected to electrophoresis on a $1 \%$ agarose gel. The DNA fragments in the size range of $5(0)-3(0) 0$ bp were collected using a DEAE membrane. The purified fragments were then ligated into the Pstl site of PUC18. The E. coli bacterial strain DH5 $\alpha$ was transformed and plated out on Xgal/IPTG/ampicillin/LB plates. Individual colonies were picked out and grown in $5 \mathrm{ml}$ of $\mathrm{LB}$ containing $10 \mu \mathrm{g} / \mathrm{ml}$ of carbenicillin. Clones containing highly-repeated sequences.were identified by transferring Pstl-digested plasmid mini-preparations from 1\% agarose gels to Hybond $\mathrm{N}^{+}$nylon membranes and hybridizing these with ${ }^{32}$ P-labelled Haclllrestricted total genomic DNA. Those giving strong signals were discarded and the remaining putative low-copy clones werc PCR-amplified.

Other clones were also employed in the study. Three of these (UGTI, UGT 159 and (UGT 185), derived from the progeny of $P$. squamulatum $\times P$. glaucum, were kindly provided by Dr. P. Orias-Akins (Tifton, Georgia). Two heterologous known-function prohes, both maintained in this laboratory, were also included in the study. The locus designated $X p s r .39 /(F h p)$ was detecled by a probe for chloroplastic fructose-1,6-bisphosphatase (Raines et al. 1988) kindly provided by T. Dyer. Another locus, designated Nra, was detecled by a nitrate reductase probe (Cheng et al. 1986) kindly provided by Prof. A. Klcinhof's.

\section{RFLP procedures}

Methods for plant DNA isolation, restriction enzyme reactions, gel electrophoresis, Southern transfer, probe labelling and filter hybridization, were as described by Sharp et al. (1988), with the modification that Hybond $\mathrm{N}^{+}$nylon membranes were used. After hybridization the membranes were washed twice in $2 \times$ SSC and $1 \%$ SDS for 15 min each at $65 \% \mathrm{C}$, followed by $\mathrm{tw0}$ washes in $(0.2 \times \mathrm{SSC}$ and $1 \%$ SDS for 15 min each at $65^{\circ} \mathrm{C}$. Membranes were stripped for re-use by adding a boiling solution of $0.5 \%$ SDS and $0.1 \times$ SSC and agitaling for $15 \mathrm{~min}$.

Initially, 20() clones, identified as single or low copy, were used to screen DNA from the 19 genotypes digested with two of four restriction enzymes, EcoRI. EcoRV. HindIII and Dral. Subsequent screening for polymorphic probe-enzyme combinations was restricted to the four parental lines involved in the two $F_{2}$ populations. The probe-enzyme combination which gave the clearest polymorphism between the respective parental genotypes was used in the mapping experiments.

\section{Linkage analysis}

The data were analyzed using the programme MAPMAKER (version 3) supplied by E. S. Lander, Whitehead Institute for Biomedical Research. Cambridge, Massachusetts. Linkage groups were first obtained using two-point analysis with a LOD score of 8 and a maximum recombination fraction of 0.20 . Multipoint analysis was then used for markers in each individual linkage group to determine their relative order with a LOD threshe' I of 2.5 . These LOD thresholds werc arrived at empirically. The scvere, two-point. L.OD discriminated pseudo-linkages due to segregation distortion. Remaining marker loci that could not be placed under these criteria were then assigned to the most-likely intervals. The data was further scrutinised to identify points for which any possible ambiguities in order existed.

\section{Results}

\section{Library}

As demonstrated in many other crop species (e.g., Burr et al. 1988; McCouch et al. 1988), the use of Pstl to gener-
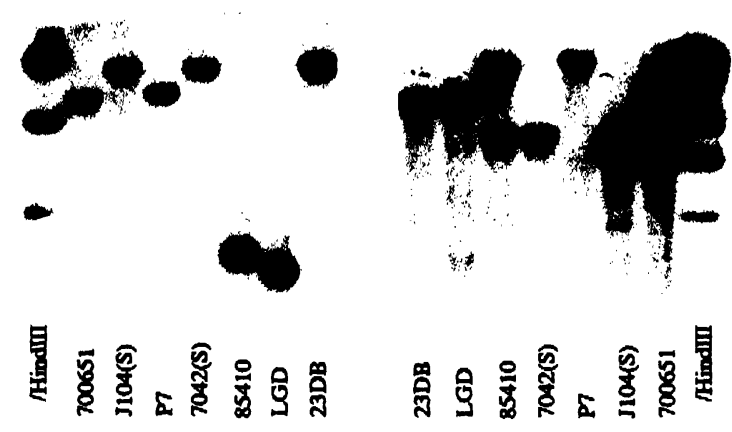

Fig. 1 Autoradiograms, derived from probing with PgPSM473, to $E$ E'oRI (left)- and EcoRV (right)-digested DNA from seven pearl millet genotypes (see text for explanation)

ate genomic DNA fragments for the production of a library enriched for low-copy clones also proved effective in pearl millet. Only $11 \%$ of the clones gave strong signals when hybridized with total genomic DNA, and these were discarded. Eighty-three per cent of the remaining clones detected single-copy sequences, as determined from hybridization patterns with (wo restriction enzymes. Eight per cent detected low (2 to 4)-copy sequences, and the other $9 \%$ detected higher copy-number sequences.

\section{Polymorphism}

Analysis of the 19 genotypes with 200 low-copy-number genomic clones indicated that a very high level of polymorphism exists in this cross-pollinated crop species. Eighty-five per cent of the clones revealed polymorphic hybridisation patterns among these 19 genotypes when digested with two restriction enzymes, with an average pairwisc polymorphism between all the probe-en $z y m e$ combinations of $56 \%$ ( $35609 / 63249$ comparisons). This percentage, of course, is highly dependent on the particular sample of genotypes chosen for examination. However, the pearl millet value was significantly higher than that found in many predominantly self-pollinating cercal crop species, including rice (McCouch et al. 1988), wheat (Chao et al. 1989; Devos et al. 1992) and barlcy (Graner et al. 1991; Heun et al. 1991), but was similar to that observed in the predominantly cross-pollinating maize (Helentjaris et al. 1985).

In some crop species, the detection of an RFLP pattern in any genotype-pair with one restriction enzyme is likely to be repeated when another restriction enzyme was used because the RFLPs result primarily from insertion/deletion events. Base substitution within restriction sites may result in different patterns with different enzymes. A survey of the RFLP observed within the sample of pearl millet genotypes and probes indicated that base changes within restriction sites were common, since in most cases either an RFLP was detected with only one of the two restriction enzymes, or the RFLPs between two genotypes digested 
with two restriction enzymes did not differ by the same size increment. For example, when probed with PgPSM473, the polymorphism between 700651 and 7042(S) was detected by EcoRI but not by EcoRV. For P7 and 7042(S) the RFLPs detected by both EcoRI and EioRV indicated different origins. $P 7$ has a larger hybridizing fragment with EcoRV and a smaller one with EcoRI (Fig. 1). Furthermore. Dral, which generated the largest mean fragment size among four restriction enzymes $(11.0 \mathrm{~kb}$ for Dral, $8.8 \mathrm{~kb}$ for HindIII, $8.5 \mathrm{~kb}$ for EcoRV and $6.5 \mathrm{~kb}$ for EcoRI), failed to detect the highest level of polymorphism (73\% for HindIII, 69\% for EcoRV. 62\% for Dral and $61 \%$ for $E(o \mathrm{RI})$, as might have been expected if insertion/delction werc the most frequent causes of RFLP.

\section{Map}

Of the 166 probes used to construct the map in the LGD $\times$ 85410 cross, 108 hybridized to single-copy sequences, 45 to two-copy sequences, nine to three-copy sequences and the remaining four to four-copy sequences. Most of the probes that hybridized to low-copy sequences detected polymorphism at only a single locus in this cross and only 12 of them detected more than one locus in different parts of the genome. Overall, 181 segregating loci were detected. and they were assigned to nine linkage groups using twopoint analysis. These included six main groups [consisting of $83(46 \%), 29(16 \%), 20(11 \%), 17(9 \%), 13(7 \%)$ and $11(6 \%)$ loci respectively]. two groups each containing two loci, and a single locus detected by probe PgPSM870). which segregated independently of any other. When a LOD $<8$ was used, a unique placement on linkage group 4 was found for one of these small doublets (containing Xpsm 464 and Xpsm409.1).

The mapping data for the large linkage group, containing $46 \%$ of the loci mapped, identified a region where some points showed close linkages but could still not be linearly ordered. To further investigate this potential translocation point, 19 loci within the group wcre also mapped in the $23 \mathrm{DB} \times$ WSIL cross. This resulted in two independent linkage groups (Fig. 2). Close scrutiny of the LGD $\times 85410$ data indicated that integrity broke down below Xpsm8.37 in linkage group 1 and above Xpsm4/6.2 in linkage group 2. The points $X p s m 6.51,672.2$ and 322 clearly lay in the intervening region but could not be assigned with confidence to either group (Fig. 3). Independence of linkage groups 1 and 2 has been confirmed in another four crosses (C. J. Liu unpublished). The final map has seven main linkage groups (Fig. 3), which presumably correspond to the seven chromosome pairs in pearl millet, and three unassigned points, including Xpsm870.

\section{Loci showing skewed allelic segregation}

Segregation distortion was very severe in the LGD $\times 85410$ cross. Eighty-six out of the one-hundred and eighty-one loci mapped in this cross deviated significantly from the expected $1: 2: 1$ or $3: 1$ segregation ratios, $29(16 \%)$ at the

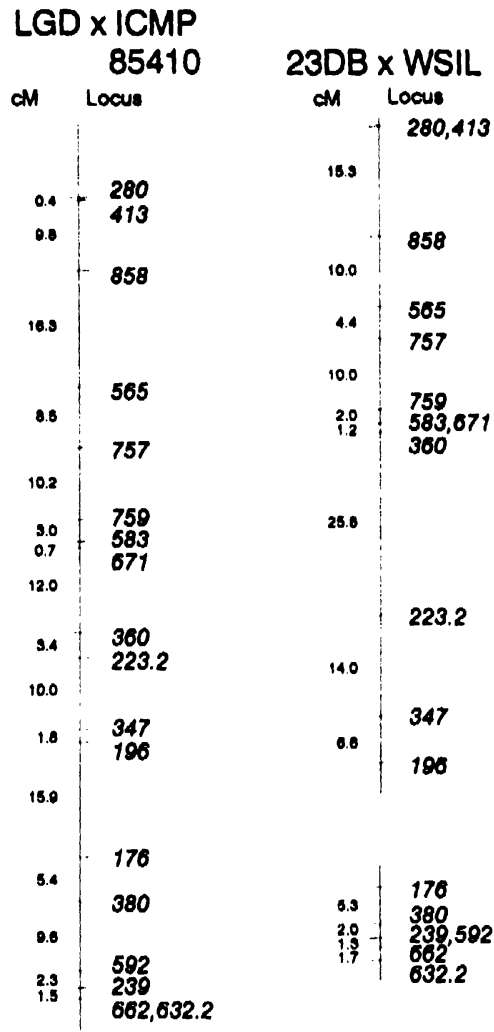

Fig. 2 A comparison of the genetic inaps for linkage groups 1 and 2 in 1 wo different crosises, 1 (.(iD)-1-B-1() $\times$ ICMP 8.5410 vs Tifi 2.31$) B_{1}$ $\times$ WSII.

$5 \%$ level of significance and $57(31 \%)$ at the $1 \%$ level. Allele frequencies could be quantified accurately only at the 45 loci showing co-dominant, i.c., 1:2:1, segregations. Of these, 19, all in the region of the putative break-point separating chromosomes 1 and 2, had normal, i.c., I:I. A:B ratios but an excess of heterozygotes (Fig. 4). The other 26 loci showed significant A:B deviations. Without exception, thesc deviations favoured alleles of the male parent ICMP 85410 and most mapped on linkage groups 4, 5 and 7 (Fig. 4).

Contrary to the findings in the LGD $\times 85410$ cross, most loci mapped in the $23 \mathrm{DB} \times$ WSIL cross showed the expected $1: 2: 1$ or $3: 1$ segregation ratios. Significant distortion was observed for only 3 out of the 19 loci, two of them (Xpsm196 and Xpsm223) at the 1\% level and the other (Xpsm347) at the 5\% level. These distortions all favoured alleles from the male parent, WSIL. and all three mapped at the bottom of linkage group 1.

The order and recombination frequencies among loci on the two different crosses

Of the 19 probes that were used for mapping in both the crosses (Fig. 2), two (PgPSM223 and PgPSM632) both de- 
Fig. 3 RFLP linkage map of the pearl millet genome. The seven major linkage groups were arbitrarily ordered from I to 7 . based on the numbers of mapped loci. Numbers on the lefi hand of bar are map dislances in centiMorgans, and /ocus names, which are on the right hand side of har, were shorlened to numbers only whenever they were detected by PgPSM probes. The two arrows indicate the region in which the breakpoint separating the first two linkage groups was located. The loci detected by single-copy clones, or low-copy clones where all copies cosegregated, are picked out in bold. The bars to the right of the loci indicate regions of possible annbiguous order; the points picked out are placed in the 'best fit' position, but could equally well be placed elsewhere within the extent of the allached vertical bur

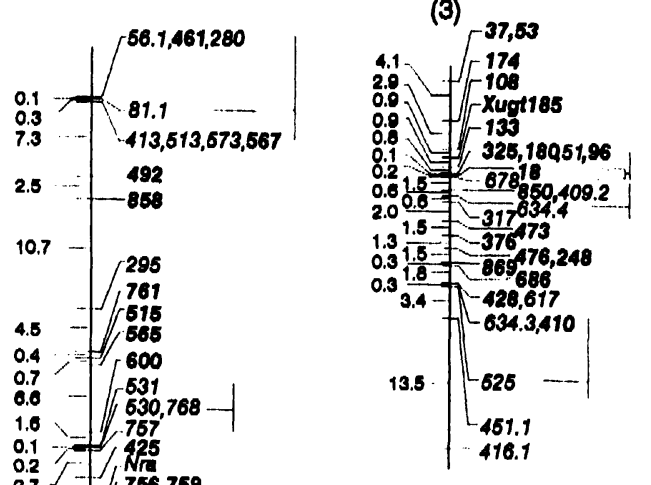

(5)

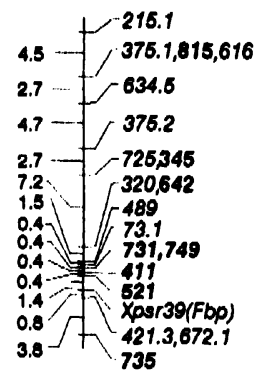

(6)

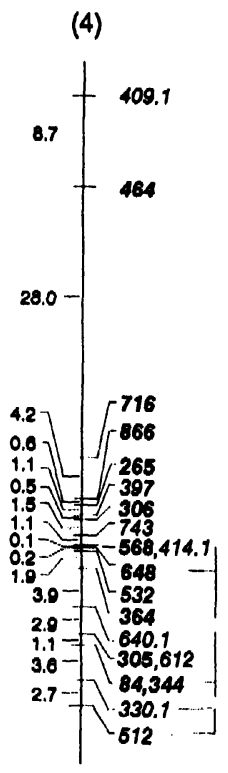

(7)

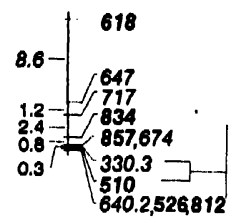

(A)

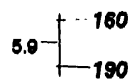

tected two loci of which one was polymorphic in 23DB $x$ WSIL. The correspondence between Xpsm223 in the latter cross with Xpsm223.I or 223.2, which lie close together in linkage group 1, is not known. The Xpsm632 locus in 23DB $\times$ WSIL is assigned to 632.2 from its location at the bottom of linkage group 2 .

The order of all loci mapped on linkage groups 1 and 2 in these two crosses was identical (Fig. 2). Compared with that in the LGD $\times 85410$ cross, there was a general reduction in recombination frequencies (and hence genetic lengths) between markers in linkage group 2 in the 23DB $x$ WSIL cross. For linkage group 1, although the distances between some points differed in the two crosses, the overall length was similar.

\section{Discussion}

\section{Scgregation distortion}

Segregation distortion is a common phenomenon in RFLP analysis and has been observed in almost all crop species studicd so far, particularly in interspecific crosses. However, few of the previous reports noted such extreme segregation deviations as was found here in LGD $\times 85410$, in intra-specific crosses, both in terms of the proportion of markers involved and the level of distortion. Moreover, all the skewed markers in our population were consistently biased toward alleles from the male parent or, in the region 
Fig. 4 Deviant loci and their distribution in the four regions of the pearl millet genome in the cross LGD. $1-B \cdot 10 \times$ ICMP 85410. Those loci scored as $3: 1$ segregations, and loci which co-segregated with the loci listed here, were not included. Key: $A$. frequency of homozygous female parental genotype; $B$. frequency of homozygous male parental genotype: $H$, frequency of heterozygous genotypes

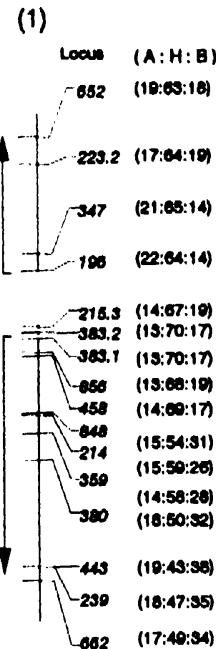

(2)
(4)

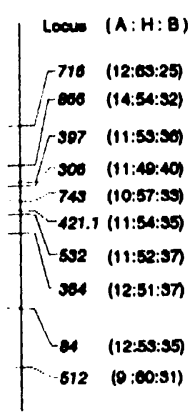

(5)

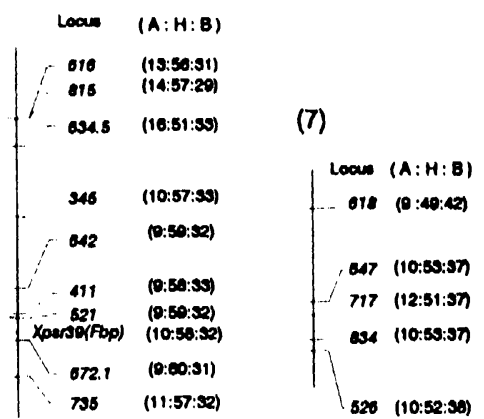

of the translocation breakpoint, towards heterozygous genotypes. None was significantly skewed towards the female parent. LGD. Such distortions can be due to gametic or hybrid selection at different developmental stages for genes affecting fitness (O'Donoughue et al. 1992). LGD is much earlier to flower, has a smaller biomass, and is generally less vigorous than ICMP 85410, probably due to the presence of the $e_{1}$ gene for photoperiod-insensitive early flowcring (Burton 1981). Not all $F_{2}$ plants produced enough tissue to be sampled, or survived to produce sceds, so alleles from the least-fit parent may have been sclected against. However, data from the 23DB $\times$ WSIL cross suggest that such extreme segregation distortion is unlikely to be a general property of this species.

\section{Chromosome evolution}

It has been widely speculated that several crop species have evolved from species with lower basic chromosome numhers. Evidence supporting this hypothesis has been found by RFLP studies in maize (Helentjaris et al. 1986; Whitkus et al. 1992), sorghum (Hulbert et al. 1990; Whitkus et al. 1992: Chittenden et al. 1994), and brassicas (Slocum et al. 1990; Kianian and Quiros 1992). It has been speculated from cytogenetical evidence that $x=5$ may be the original basic number in the genus Pennisetum, and that the apparently diploid species, $P$. glaucum, with $2 n=14$ chromosomes, may be a "secondary diploid having a secondary balanced number of $x=7$ " (Jauhar 1968, 1970, 1981). If duplication of part of the original genome has been involved during the evolution of pearl millet, some duplicated loci should be consistently located together in different regions of the present genome.

Twelve out of the one-hundred and fifty-eight probes allowed detection of more than one locus in the LGD $\times$ 85410 cross. Figure 3 shows that duplicated loci detected by the same probe. i.e., those with decimalised postscripts, can be adjacent. such as those detected by PgPSM223 (linkage group 1) and PgPSM38.3 and PgPSM227 (linkage group 2), be far apart, or even on different linkage groups. such as those detected by PgPSM330) (linkage groups 4 and 7) and PgPSM640) (linkage groups 4 and 7). Some of the loci, which were detected by the same low-copy-number sequences but which mapped on separate chromosomes. showed evidence of non-random distribution. For example, clones PgPSM421, PgPSM634 and PgPSM215 detected copies, in the same order, on linkage groups 2 and 5, and both PgPSM330 and PgPSM640 detected copies on linkage groups 4 and 7 . Confirmation of this possible ev. idence for duplicated regions of the genome must await more extensive data.

The proportion of single-copy sequences mapped in LGD $\times 8541$ () (110 out of 166 ) was lower than the $83 \%$ found in the low-copy-number sample in the genomic library, simply because there is a greater chance of detecting polymorphism with probes which hybridize with multiple copies. While sequences present in several copies are useful for map construction, for studies of chromosome evolution, and in some fingerprinting applications, unambiguous single-copy sequences are easier to apply in experiments in which the map is to be transferred to other crosses; for example, to tag genes of agricultural importance. For this reason, single-copy sequences, and multiple-copy sequences where all copies have been shown to cosegregate at a single locus, and so are likely to provide unambiguous markers in further applications, have been highlighted in Fig. 3.

\section{Marker clustering}

Previous studies using physical markers, such as NORs, C-bands and in-situ sites, all indicated that recombination 
is predominantly confined to the distal regions of chromosomes in Triticeae species (Linde-Laursen 1982; Dvorak and Chen 1984; Snape et al. 1985; Lawrence and Appels 1986; Wang et al. 1991). Clustering of RFLP loci around the centromeres has been repeatedly observed in several crop species. In hexaploid wheat, for which the centromere locations can be precisely located using aneuploid genetic stocks, such clustering effects are particularly obvious (Chao et al. 1989; Devos et al. 1992). Although the RFLP map generated in $P$. glaucum is relatively dense, with an average distance of $2 \mathrm{~cm}$ between markers, specific chromosome areas with tightly-linked markers are still evident. In the absence of physical markers it is not known if this is because the regions have a reduced recombination or a particularly high level of polymorphism. The clusters were not confined to one single region in each of the seven main linkage groups. For example, linkage group 1 contains at least five of these clusters.

\section{The relationship between genome size and map length}

The pearl millet map obtained in this study spans $3(03 \mathrm{cM}$. Assuming that linkage group $\mathrm{A}$ and the independent locus $X$ psm 870 each represent an additional $50 \mathrm{cM}$, we estimate that the total genome genetic length of pearl millet revealed with the present data set would be about $4(0) \mathrm{cM}$, which is the shortest in all the major crop species studied so far. For example, the map size reported for barley was between 1096 and $1453 \mathrm{cM}$ (Graner et al. 1991: Heun et al. 1991) and for rice was $1389 \mathrm{cM}$ (McCouch et al. 1988), although the nuclear DNA content for the latter is only one quarter that of pearl millet (Bennett 1976). It is well known that one of the main factors which influences the actual map size obtained in a given species is the cross used for study. The wider the cross, the smaller the total map distance is likely to be. Many RFLP maps reported so far are based on data derived from a single, often wide, cross. Thus it will not be surprising if later studies extend existing map distances. However, several different crosses, all involving adapted accessions of $P$. glaucum, have now been investigated in this laboratory. All show similar linkage intervals where direct comparisons can be made and thus we conclude that the LGD $\times 85410$ map is typical of the species.

The small map size of pearl millet will facilitate its use in some applications, but hinder progress in others. To locate genes in crosses other than those for which RFLP maps exist, a skeleton map often needs to be transferred. In pearl millet less than 40 probes will produce such a map, with an average map distance of less than $10 \mathrm{cM}$ between markers. In contrast, a larger number of probes will be needed in other crop species. On the other hand, the low levels of recombination will increase linkage drag in, for example, the transfer of genes by backcrossing. Application of the map to identify flanking recombinants, and so improve the efficiency of the backcrossing process, is thus likely to be particularly apposite in pearl millet.
Acknowledgements Financial support from the Plant Sciences Research Programme of the Overseas Development Administration. UK. administered by the Centre for Arid Zone Studies. University of Wales, Bangor, is gratefully acknowledged. We are also grateful to Rémy Maufrand (University of Wales) and M. N. V. Ratnaji Ruo (ICRISAT) for their technical assistance. A set of 47 clones which span the existing pearl millet map are available from Dr. $\mathbf{M}$. D. Gale, and all of the remaining probes are available on specific request.

\section{References}

Bennett MD (1976) DNA amount, latitude, and crop plant distribution. Environ Exp Bot 16:9,3-108

Brunken JN (1977) A systematic study of Pennisetum sect. Pennisetum (Gramineae). Am J Bot 64:161-176

Burr B. Burr FA. Thompson KH. Albertson MC, Stuber CW (1988) Gene mapping with recombinant inbreds in maize. Genetics $118: 519-526$

Burton GW (1981) A gene for early maturity and photoperiod-insensitivity in pearl millet. Crop Sci 21:317-..318

Chao S. Sharp PJ, Worland AJ, Warham EJ, Koebner RMD, Gale MD (1989) RFL.P-based genetic maps of wheat homocologous group-7 chromosomes. Theor Appl Genet 78:495-504

Cheng CL., Dewdney J, Kleinhof's A (1986) Cloning and nitrate induction of nitrate reductase mRNA. Proc Natl Acad Sci USA 83:6825-6828

Chittenden LM, Schertz KI; Lin Y-R. Wing RA, Paterson AH (1994) RFLP mapping of a cross between Sorghum bicolor and S. propinquum, suitable for high-density mapping. suggests ancestral duplication of Sorghum chromosomes. Theor Appl Genet 87: 925-933.

Devos KM, Atkinson MD, Chinoy CN, L iu C.J, Gale MD (1992) RFLP-based genetic map of the homoeologous group- 3 chromosomes of wheat and rye. Theor $\Lambda$ ppl (ienet 8.3:9.31-9.39

Dvorak J, ('hen KC. (1984) Distribution of nonstructural variation between wheat cultivars along chromosome arm $6 \beta$ : evidence from the linkage map and a physical map of the arm. Genetics 106:325-333

Graner A. Jahoor A. Schondelmaier J, Siedler H. Pillen K. Fischbeck G. Wenzel G, Herrmann RG (1991) Construction of an RFLP map of barley. Theor Appl Genet 83:250-256

Helentjaris T, King (j, Slocum M. Siedenstrang C. Wegman S (1985) Restriction fragment polymorphisms as probes for plant diversity and their development as tools for applied plant breeding. Plant Mol Biol 5:1(19-118

Helentjaris 'T, Slocum M, Wright S, Schacfer A, Nienhuis J (1986) Construction of genetic linkage maps in maize and tomato using restriction fragment length polymorphisms. Theor Appl Genet 72:761-769

Heun M, Kennedy AE. Anderson JA, Lapitan NLV, Sorrells ME, Tanksley SD (1991) Construction of a restriction fragment length polymorphism map for barley (Hordeum vulgare). Genome 34:437-447

Hulbert SH, Richter TE, Aztell JD, Bennetzen JL (1990) Genetic mapping and characterization of sorghum and related crops by means of maize DNA probes. Proc Natl Acad Sci USA 87: $4251-4255$

Jauhar PP (1968) Inter- and intra-genomal chromosome pairing in an interspecific hybrid and its bearing on basic chromosome number in Pennisetum. Genetica 39:36()-370)

Jauhar PP (1970) Haploid meiosis and its bearing on the phylogeny of pearl millet, Pennisetum typhoides Stapf et Hubb. Genetica 41:532-540

Jauhar PP (1981) Cytogenetics and breeding of pearl millet and related species. Progress and topics in cylogenetics, vol 1. Alan R Liss. New York. pp 1-289 
Kianian SF. Quiros CF (1992) Generation of a Brassica oleracea composite RFLP map: linkage arrangements among various populations and evolutionary implications. Theor Appl Genet 84: 544-.554

Lawrence GJ. Appels R (1986) Mapping the nucleolus organiser. seed protein loci and isozyme loci on chromosome IR in rye. Theor Appl Genet 71:742-749

Linde-Laursen I (1982) Linkage map of the long arm of barley chromosome $3 \mu$ sing $C$-bands and marker genes. Heredity 49:27-35

McCouch SR. Kochert G. Yu ZH. Wang ZY. Khush GS. Coffman WR, Tanksley SD (1988) Molecular mapping of rice chromosomes. Theor Appl Gene1 76:815-829

Minocha JL (1991) Pearl millet cytogenetics. In: Ciupta PK and Tsuchiya T (eds) Chromosome enginecring in plants: genetics, breeding, evolution (Part A) Elsevia. Amslerdam. pp 599-611

O'Donoughue IS. Wang Z. Röder M. Kneen B. Leggell M. Sorrells ME. Tanksley SD (1992) An RFLP.based linkage map of oats based on a cross between two diploid taxa (Aiena allantic a $\times A$. hirtula). Genome 35:765-771
Raines CA. Lloyd JC. Longslaff M. Bradley D. Dyer T ( 1988 ) Chloroplast fruclose-1,6-biphosphatase: the prexduction of a mosaic gene. Nucleic Acids Res 16:79.31-7942

Sharp PJ. Kreis M. Shewry PR. Gale MD (1988) loculion of $\beta$-amylase sequences in wheal and its relatives. Theor Appl Genet $75: 286-290$

Slocum MK. Figdore SS. Kennard WC. Suzuki JY. Osborn TC (1990) Linkage arrangement of restriction fragment length polymorphism loci in Brassica olerecea. Theor Appl Cienet 80:57-64

Snape JW. Flavell RB. O' Dell M. Hughes WG. Payne PI (198.5) Intrachromosomal mapping of the nucleolar-organiser region relative In three marker loci on chromosome IB of wheall (Triticum at'stilum). Theor Appl Genel 69:26.3-270

Wang MI., Atkinson MD. Chinoy (N. Devos KM. Liu (J. Rogers WJ. Cale MD (|99|) RILLP-based map of rye (S. cereale') chromosome IR. Theor Appl (ienet 82:174-178

Whitkus R. Docbley J. Lee M (1992) Comparative genome mapping of sorghum and inaize. (ienetics 1.32:1119-11.31) 